\title{
ALTERNATIVE ANALYSES OF LOCOMOTIVE STRUCTURAL DESIGNS FOR CRASHWORTHINESS
}

\author{
Eloy Martinez \\ David Tyrell \\ Volpe National Transportation Systems Center \\ US Department of Transportation \\ Cambridge, MA 02142 USA
}

\begin{abstract}
Enhanced crashworthiness performance of North American locomotives is proposed by both increasing the design loads on specific structural components or by describing the crashworthiness performance under specific impact conditions. The design loads for a conventional North American locomotive and the description of performance are intended to provide the same level of crashworthiness protection. A generic design was developed to illustrate the types of calculations that can be used to show that the design loads can be supported and the desired level of performance can be met. The performance of the generic locomotive subjected to a collision with a grade crossing object is measured by its ability to prevent penetration of the object into the occupied volume where the crew rides out the collision. The closed form hand calculations apply established structural analysis limit load theory and show the design loads can be supported. The finite element analysis results capture large deformation non-linear behavior and show the desired level of performance is achieved.
\end{abstract}

\section{INTRODUCTION}

The Locomotive Crashworthiness Working Group of the Federal Railroad Administration's Railroad Safety Advisory Committee (RSAC) is currently developing recommendations on locomotive crashworthiness. The RSAC is a government/ industry committee including all segments of the rail community, with the purpose of developing recommended solutions to safety issues.

Previously, as part of the Working Group activities, a study was conducted of baseline and modified locomotive crashworthiness performance in selected collision scenarios [1]. A series of collision scenarios, intended to bound the range of locomotive collisions that occur, were defined. Using these collision scenarios, with designs representative of current locomotives, baseline levels of occupant protection were determined. Numerical analyses were conducted using both dynamic, non-linear, large deformation, finite element modeling techniques as well as rigid body collision dynamic modeling techniques to assess levels of crashworthiness protection. Design modifications were investigated and compared against baseline designs. This information was then presented to the Locomotive Crashworthiness Working Group of RSAC to assist in the development of recommendations.

The Working Group is currently considering alternative means of specifying crashworthiness: with design loads and with descriptions of performance under impact conditions. This paper describes some of the work that has been performed for consideration by the Working Group in evaluating those alternatives. The Working Group has not yet finalized its recommendations.

Design loads are applied to the structure at particular points without the structural component experiencing yielding or surpassing its ultimate capacity. The descriptions of performance under impact conditions are defined by a set of collision scenarios that the design has to withstand by preventing intrusion into operator/crew occupied space and limiting deceleration pulses in those same volumes. Meeting either the design load or the description of performance is determined by conducting: full and/or scaled static/crash tests, hand calculations, detailed numerical modeling, or some combination of these techniques.

This paper includes descriptions of the analyses suitable for showing that both the design loads and performance descriptions are met. It is understood that there are other techniques applicable for demonstrating compliance such as full and/or scale testing of single components or complete structural systems, but the details of using these techniques are outside the scope of this paper. 
During the review process of collision scenarios, two classes were examined, in-line and oblique $[1,2,3]$. Depending on the point of initial impact during the collision different structural components are engaged and hence recommendations are being considered for: the wide-nose short hood, collision posts, and the window structure in the cab area. The purpose of this paper is to present a comparison of the predicted response from a generic locomotive design using both design loads and a description of performance. Only one collision scenario is considered in this paper - an in-line collision, in which the locomotive is impacted above its main structure.

\section{DESIGN LOADS}

In the event that override has occurred in an in-line collision the key structural elements that resist the intrusion of an object into the occupied volume are the collision posts. Therefore, these structural elements should withstand a number of loads applied at various heights and directions. Under consideration are the following design loads for locomotive collision posts:

Each locomotive must be equipped with at least two collision posts or equivalent structures on the short hood end located approximately at the 1/3 points across the locomotive width.

Each collision post must be of sufficient height to extend within 9 inches of the height of the bottom of the operating cab window frame and must withstand the following loads without exceeding the ultimate strength of the posts and their attachments to the underframe:

(1) A 750, $000 \mathrm{lb}$. longitudinal load applied at the base of each collision post, at any angle in the horizontal plane in the range of $+/-15$ degrees of the longitudinal axis of the locomotive,

(2) A 500,000 lb. longitudinal load applied on each collision post at a height 30 inches above the top of the underframe, at any angle in the horizontal plane in the range of $+/-15$ degrees of the longitudinal axis of the locomotive,

(3) Any longitudinal load $(F)$ :

a) that is applied at a vertical location greater than 30 inches above the top of the underframe up to the top of the collision post, and

b) which develops the same moment at the base as a $500,000 \mathrm{lb}$. load applied at 30 inches above the underframe, and

c) that is applied at any angle in the horizontal plane in the range of $+/-15$ degrees.

Figure 1 depicts the top and side views of the locomotive and how the loads are applied for clarification of the text. The outof-plane loads are applied to assure that the collision post performs well in the event of loading conditions where lateral torsional buckling dictates the response of the collision post.

The first statement is assumed to be met, with a pair of collision posts for this design located at the $1 / 3$ points across

the locomotive width. The loads to be applied to the posts cannot exceed the ultimate strength of the post and the connection must be able to transmit the loads without failure.
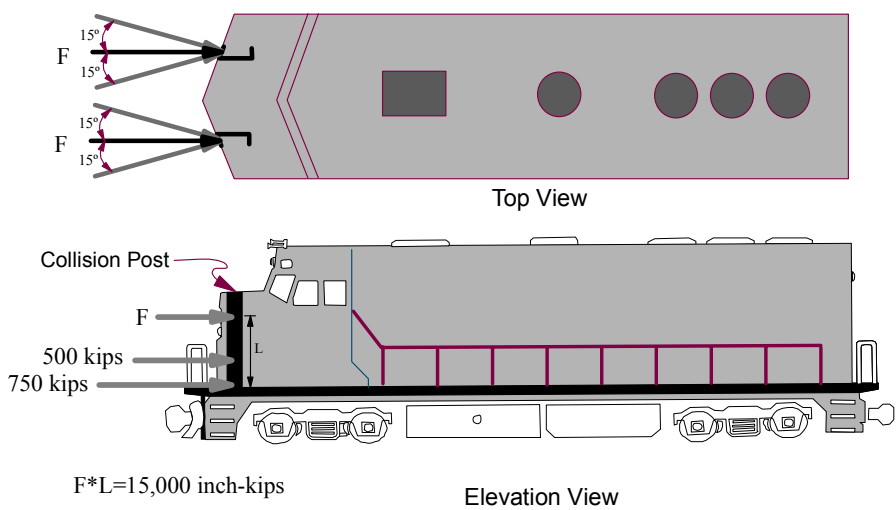

Figure 1. Design Loads for Collision Posts

The cross-sectional shape and the relative position of the collision post on the sub deck are depicted in Figure 2. An I section was chosen due to the need for torsional resistance caused by the out of plane loads of $+/-15^{\circ}$. The dimensions are defined as follow: $d$, the depth of the web of the beam, is equal to 16.0 inches, $b$, the width of the flange, is 8.0 inches, $t_{w}$, the thickness of the web, is 1.5 inches, and finally $t$, the thickness of the flange, is 1.5 inches.

(a)

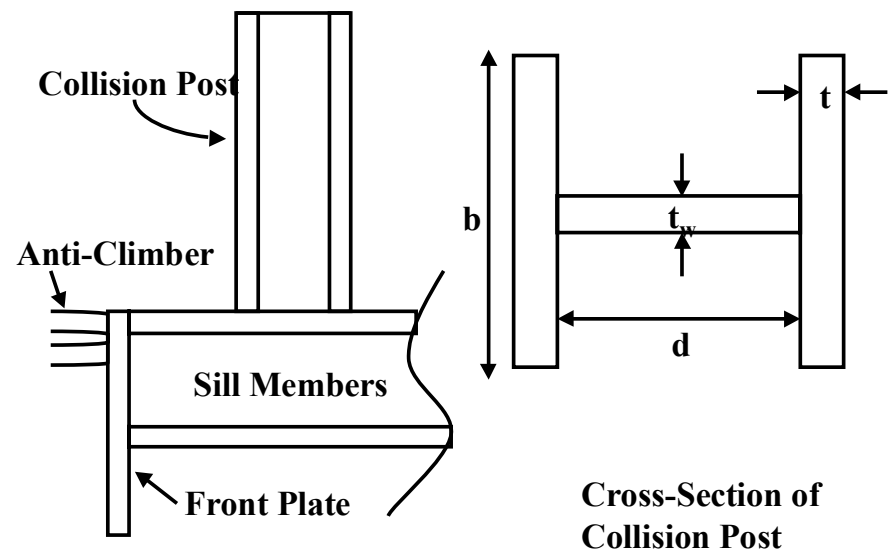

Figure 2. Position and Cross-section of Collision Post Analyzed

The post is constructed from a steel which has the following nominal material properties: $\sigma_{y}=50 \mathrm{ksi}, \sigma_{u}=72 \mathrm{ksi}, \varepsilon_{f}=0.21$, where $\sigma_{y}$ is the yield strength, $\sigma_{u}$ is the ultimate strength, and $\varepsilon_{f}$ is the ultimate strain to fracture. It is assumed that collision post is fully fixed to the deck structure and underframe so that all loads applied to the collision post are resisted by the bending stiffness of the underframe. 


\section{Shear Calculations - Load Case (1)}

The first load case, a 750,000 lbs. force applied at $+/-15^{\circ}$ is a shear load. The shear distribution in an I-section for a load applied longitudinally is linearly increasing in the flanges with a maximum value in the flange at the intersection of the web and flanges. This value is far smaller than the resistance provided by the web which varies parabolically from the top to the bottom and has a maximum value at the neutral axis of the section [4]. The maximum applied shear stress experienced for the longitudinal load is calculated by using the following expression:

$$
\tau_{\text {applied }}=\frac{V}{A_{\text {web }}} .
$$

The maximum shear stress calculated for the longitudinally applied load is $31.3 \mathrm{ksi}$. Using a von Mises failure criterion for the material, the maximum shear resistance of the material is calculated as $41.6 \mathrm{ksi}$ using the following expression:

$$
\tau_{\max }=\frac{\sqrt{3}}{3} \sigma_{u}
$$

Therefore the size of the cross-section is sufficient to withstand the load. Next a check is made for the load applied through the $15^{\circ}$ angle. The load is split into its respective components of shear in the longitudinal and lateral directions and equation (1) is applied to the respective load. The area used for the lateral shear resistance is twice the area of a single flange. The resultant shear stress is $38.3 \mathrm{ksi}$ which is less than the maximum shear resistance.

\section{Bending Calculations - Load Case (2)}

The second load case, a 500,000 lbs. force applied 30 inches above the deck in the range between $+/-15^{\circ}$ is a bending load. The out-of-plane loading condition will cause either torsional displacements and stresses or unsymmetrical bending deformations and stresses depending upon the manner in which the load is applied. The fully plastic torsional capacity of this post is calculated using the following expression [5]:

$$
T_{p}=\tau_{\max } t^{2}\left(\frac{d+2 \cdot t}{2}+b-\frac{7}{6} \cdot t\right)
$$

where $\mathrm{t}$ for this section is the constant thickness of both the web and flanges. The maximum torque is 1474 kip-in. To check this against the 500 kip load at 30 inches the fully plastic torque is converted into an equivalent load acting through an eccentricity. The eccentricity is calculated as the lateral edge length of the triangle formed, $l_{\text {lat }}$, when the load acts at $15^{\circ}$ measured from the longitudinal axes of symmetry to the neutral axis of the section. The longitudinal edge length, $l_{\text {long, }}$, is one half the height of the web plus one flange width. The length is calculated using the following expression:

$$
l_{\text {lat }}=\tan \left(15^{\circ}\right) \cdot l_{\text {long }} .
$$

The eccentricity calculated is 2.55 inches and the resulting load capacity of the section is 578 kips which is greater than 500 kips required.

An alternative solution is to assume that the load is applied through the shear center of the section so that only bending deformations exist without twisting deformations. This is the case of unsymmetrical bending. The relation between the angle of load inclination, $\varphi$, measured positive from the $\mathrm{x}$-axis and which passes through the shear center, and the angle at which the neutral axis is inclined, $\alpha$, is given by [5]:

$$
\tan \alpha=\frac{I_{x y}-I_{x} \cdot \cot \varphi}{I_{y}-I_{x y} \cdot \cot \varphi},
$$

where $I_{x y}$ is the product moment of inertia of the section, $I_{x}$ is the bending moment of inertia about the major axis, and $I_{y}$ is the bending moment of inertia about the minor axis. The section chosen is doubly symmetric so the product moment of inertia is zero. Using this relationship as well as the fact that the maximum plastic moment capacity of the beam is calculated from [5]:

$$
M_{p}=\frac{A_{\mathrm{sec} t}}{2} \cdot d^{p} \cdot \sigma_{o},
$$

where $M_{p}$ is the plastic moment of the section about the neutral axis, $A_{\text {sect }}$ is the structural shapes cross-sectional area, $d^{p}$ is the distance between compressive and tensile centroids, and $\sigma_{o}$ is the equivalent flow stress of the material. The equivalent flow stress also can be determined from the stressstrain curve [6]:

$$
\sigma_{o}=\sqrt[3]{\frac{\sigma_{y}^{2} \sigma_{u}}{1+n}}
$$

where $\sigma_{u}$ is the ultimate strength of the material and $n$ is the exponent of the power law representation of the stress-strain curve, $\sigma=A \varepsilon^{n}$, with typical values for the steels used in rail applications ranging between 0.1 and 0.2. An approximate expression for the flow stress is given for ease of computation:

$$
\sigma_{o}=\sqrt{\sigma_{y} \sigma_{u}}
$$

One can calculate the maximum load that the section can withstand using the following expressions [5]: 


$$
\begin{aligned}
& P_{\max }=\frac{M_{p}}{L}, \\
& P_{\max }=\frac{M_{p}(l+a)}{a \cdot(l-a)},
\end{aligned}
$$

where $L$ is the moment arm of the load applied on the cantilever beam, 30 inches, $l$ is the height of the collision post, and $a$ is the distance measured from the top of the collision post to the point of load application. Equation 9a is applicable to a collision post with the following boundary conditions, fully fixed at the level of the sub-base and free at the tip. Equation $9 \mathrm{~b}$ is applicable to a fully fixed condition at the level of the sub-base and a pinned condition at the level of the roof of the short hood.

For the angled load, it is assumed that the load is applied to one collision posts and the short hood ties the two posts together providing additional restraint at the top. Consequently, equation (9b) is used for the angled load application. For the longitudinal load, it is assumed that the loads are applied simultaneously to both collision posts, reducing the top restraint on the posts. Consequently equation (9a) is used when determining the ultimate plastic capacity of the post loaded longitudinally.

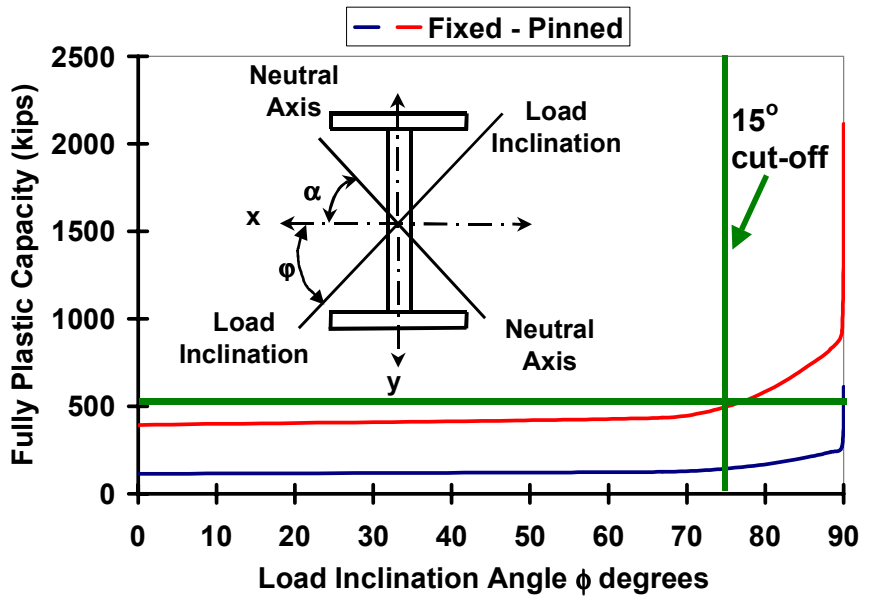

Figure 3. Variation in Plastic Capacity with Initial Load Inclination

Figure 3 summarizes the results from a series of these calculations conducted to determine the variation in fully plastic capacity with changes in the inclination of the applied load. When the post is loaded in the longitudinal direction it has a plastic bending capacity of 612 kips. When loaded in the lateral direction the plastic bending capacity is 114 kips. As the load is applied through small angle changes, up to the $15^{\circ}$ angle, the plastic capacity drops off significantly. Since the hood now supports the top of the collision post the second equation is used and the capacity at $15^{\circ}$ is 501 kips. Small changes in load inclination greatly effect the inclination of the plastic neutral axis and hence the capacity of the post. After the $15^{\circ}$ cut-off is reached the plastic neutral axis changes very slowly and is close to that of the sections minor bending capacity. The actual response of the beam lies somewhere between the fully torsional capacity and the fully plastic bending capacity.

\section{Bending Calculations - Load Case (3)}

The last load was stipulated to ensure that the collision post cross-section remains sufficiently large to resist an equivalent moment as generated by that from load case (2). Since the cross-section of the generic collision post presented remains the same as opposed to tapering that resistance is maintained and no further calculations are warranted.

\section{DESCRIPTION OF PERFORMANCE}

The description of performance includes a locomotive impact with a proxy object at a prescribed speed for which there must effectively be no intrusion into the operator's cab. Like the design loads, the description of performance is intended to assure protection of the operator in the event that override has occurred in an in-line collision.

\section{Front End Structure (Collision Posts)}

(1) Objective: The front end structure of the locomotive (i.e. in traditional locomotive design, that end containing the short hood, collision posts, and locomotive cab) shall withstand a frontal impact with a proxy object which is intended to simulate a tank car.

(2) Proxy Object Characteristics and Orientation: The proxy object shall have the following characteristics: cylindrical shape; 48-inch diameter; 126 inches in length; 65,000 pounds in weight; and uniform density. The longitudinal axis of the proxy object shall be oriented perpendicular to the longitudinal axis of the locomotive.

(3) Impact and result: The front end structure shall withstand a 30 mile per hour impact resulting in no more than 24 inches of crush along the longitudinal axis of the locomotive. The center of impact shall be 30 inches above the top of the locomotive underframe along the longitudinal centerline of the locomotive.

Figure 4 clarifies the collision scenario described by the text above. The centroid of the rigid proxy object is located 30 inches above the deck of the locomotive. By defining both a mass and an initial speed the specification is dictating what initial kinetic energy is present in the collision. Next by defining the maximum allowed crush distance, the specification dictates the energy absorption that the short hood and collision posts must provide. 


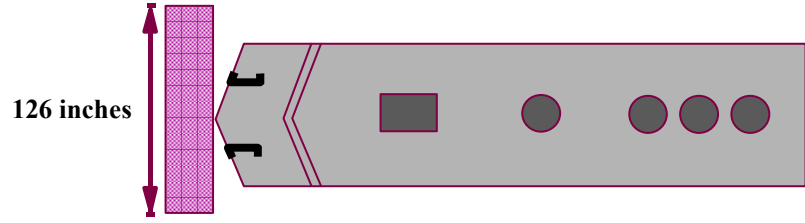
$\mathbf{V}_{\mathbf{0}}=\mathbf{3 0} \mathbf{m p h}$

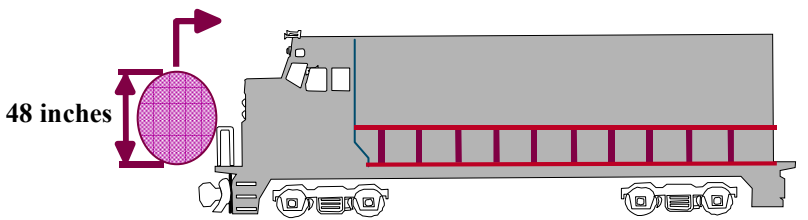

Figure 4. Schematic Illustration of Crashworthiness Performance in Override Collision Scenario

\section{Finite Element Methodology}

The crush characteristics of the generic locomotive subjected to an override collision scenario are determined by using a dynamic, non-linear, large deformation finite element model. Currently there are several commercial codes used to conduct these types of analyses, such as LS-DYNA 3D [7], ABAQUS Explicit [8], and PAM CRASH [9]. The use of an explicit finite element code is suggested due to the following factors; collision events are dynamic impact events, there are very complex interactions during the course of the impact event where contact conditions vary widely, as components fail very complicated post-buckling responses must be accurately tracked, and there are large material, geometrical, and combined non-linearities where it may be necessary to model material response with degradation and failure. In developing a robust finite element model, there are five areas which must be addressed: description of geometry, choice of mesh refinement and element formulation, characterization of material properties, definition of loads, initial, and boundary conditions, and the choice of solution formulation and desired output information.

\section{Model Simplification}

The first step is to simplify the geometry of the structure to be modeled. This includes whether spot welds or continuous welds and bolted connections need to be included in the model. A common practice is to generate a perfectly seamless connection by using coincident nodes at weld locations. This simplification will increase the overall stiffness of the structure. However, it may also decrease significantly the number of elements needed in the model and thus still capture global behavior without the computational expense. If the connections are of particular concern detailed models using sub-structured inputs from a global analysis may assist in determining the quality of the simplification and the connection.

Areas with cut-outs will significantly affect predicted response due to localized stress raisers. Current practice requires that any loss of stiffness associated with cut-outs be regained by designing stiffeners and reinforcement at the location of the cut-out. The modeling of fillets at connections can adversely affect computational time due to the need of mesh refinement in the area of the fillet and sufficient transition areas to coarser element sizes. Similar to the weld and connection analyses it may not be necessary to model the fillet as the trends in global behavior may not be affected by the local effects. Later checks should be conducted using either additional finite element models or hand calculations to determine if the affects of these type of refined details in the structure cause global changes in response due to premature failure by tearing/fracturing or locally buckling.

The model developed for the wide nose short hood with a pair of collision posts located at the one-third points is simplified by assuming the welds as seamless. No cut-outs are modeled and the connection to the sub-base is defined as perfectly rigid and hence the sub-base geometry is also not modeled. This assumption is made because the stiffness of the sub-base is significantly larger than that of the super structure. The maximum allowed crush distance is 24 inches which is sufficiently small that the cab structure was not modeled. For greater crush distances, the cab should be modeled due to the interaction between the hood material and the front face of the cab. Due to the symmetry of the system a one-half model is constructed and the calculated force crush response is doubled to account for both halves.

This model is appropriate for evaluating the crush behavior of the collision posts and short hood. The main structure of the locomotive and the attachment of the collision posts and short hood to that structure require additional analyses in order to assure that the main structure can support the load associated with the crush of the collision posts and short hood.

\section{Mesh Refinement and Element Formulation}

The second step in model development is choosing appropriate element formulations with section properties. For the large sheet structure of the wide nose short hood, a shell formulation is capable of capturing the folding/crushing response during impact events. It is absolutely necessary to conduct a series of analysis with progressively finer meshes to determine that the solution obtained is adequately converged. Naturally depending on the element formulation chosen displacement and/or stress convergence may vary. Quadrilateral reduced integration 4 noded shells were used to model the hood and posts. The wide nose short hood was split into three components, the roof, the front facing plates, and the side-walls. Each short hood component is constructed with 1940, 1890, and 1564 elements respectively. The sheet thickness is 0.375 inches for all three components. The collision post is constructed with 1428 elements. The rigid object is constructed from 2750 elements. Figure 5 shows the model with mesh used for the analyses. 


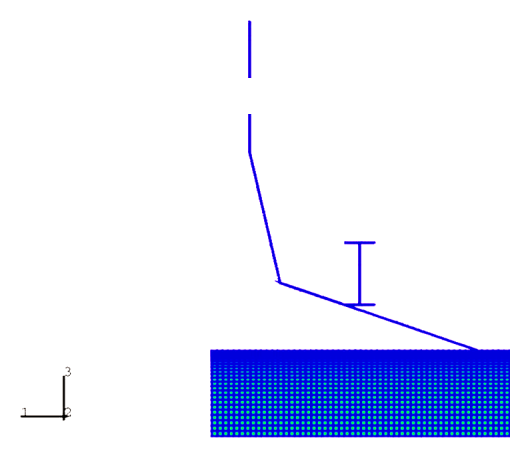

Top View

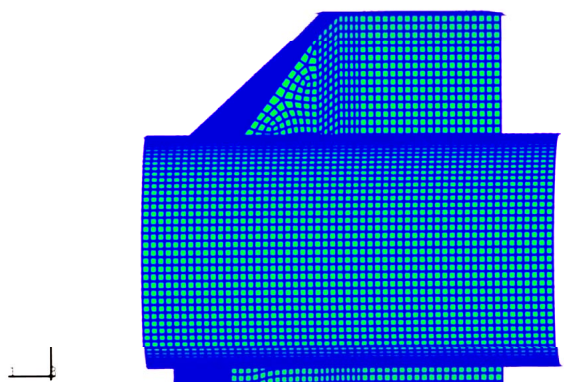

Front View
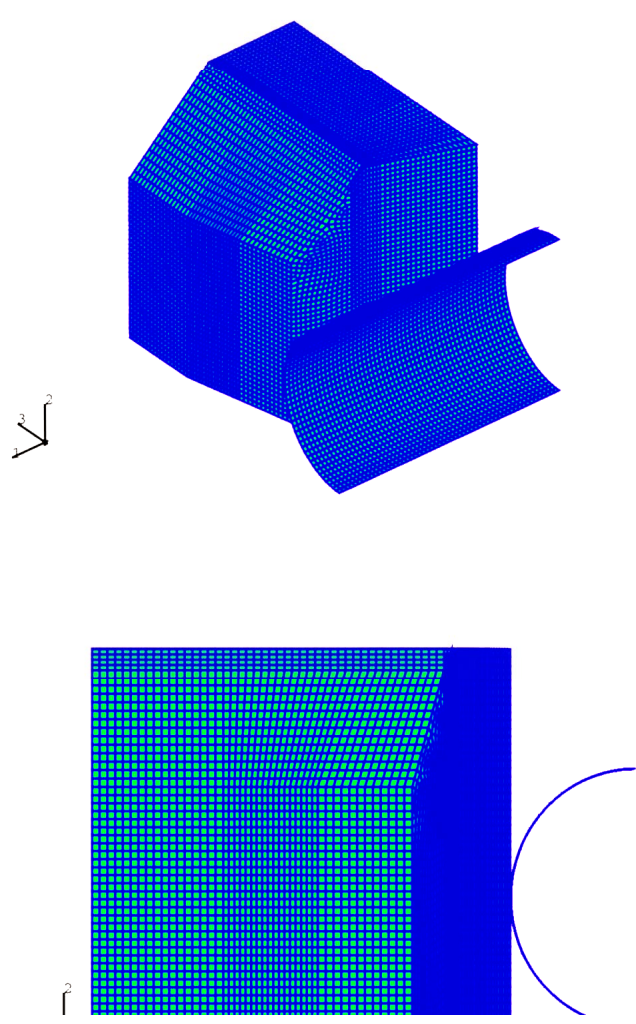

Side View

Figure 5. Finite Element Model Used in Analyses

\section{Material Characterization}

The next step is to characterize the material properties. For very high impact speeds (hundreds of feet per second) material strain rate effects may be important, however, for these analyses these effects are of second order importance and not modeled. The material commonly used in the design of locomotive short hoods is a mild steel. During dynamic crush, this material behaves in an elastic plastic manner with kinematic hardening. After sufficient plastic strain is achieved, the material degrades and softens with eventual failure by tearing and/or fracture.

The actual post is constructed from an ASTM Grade A $50 \mathrm{ksi}$ material which has the following nominal material properties: $\sigma_{y}=50 \mathrm{ksi}, \sigma_{u}=72 \mathrm{ksi}, \varepsilon_{f}=0.21$, where $\sigma_{y}$ is the yield strength, $\sigma_{u}$ is the ultimate strength, and $\varepsilon_{f}$ is the ultimate strain to fracture. Most explicit finite element codes require that the stress strain information be entered using true stress and true strain as opposed to engineering stress and engineering strain. This choice of the stress and strain measure is due to local effects during tensile testing where necking occurs and the cross-sectional area decreases, but the area used to determine engineering stress and strain remains constant and so the stress-strain diagram exhibits softening behavior. The nominal values were converted to true stress and strain measures and incorporated into the model.

\section{Initial and Boundary Conditions}

Next, the loads, initial conditions, and boundary conditions are defined. Referring to Figure 1 and Figure 5, the load is applied by defining an initial velocity to the rigid object which represents a steel cylinder. The rigid object is raised above the sub-base 30 inches and is wider than the locomotive. The path of the rigid object is restrained to motion in the longitudinal direction of the locomotive solely. The collision posts are modeled as perfectly welded to the roof and front plates of the hood. As mentioned earlier due to the relative difference in stiffness between the short hood and the underframe, the underframe geometry is not modeled explicitly, instead the nodes at the base of the short hood and collision posts are assigned fully fixed boundary conditions. The rear roof and side-walls are not assigned any support conditions above the sub-base to simulate a free floating hood. The penetration distance is defined as 24 inches, so the actual cab structure is not modeled. Symmetry conditions are imposed at the centerline of the hood. The weight assigned to the steel coil is 65,000 lbs. 


\section{Solution Formulation and Requested Output Information}

The solution formulation chosen for the finite element model was that of an explicit code. The information requested as output are the stresses, strains, displacements, velocities, accelerations, and reaction forces on the short hood. Displacement, velocity, and acceleration of the reference node on the rigid body is also requested. In order to determine the quality of the solution obtained, the strain energy, internal energy, kinetic energy, plastic dissipation, artificial energy (also called hourglass energy), and total energy are requested.

\section{Finite Element Results}

The generic wide nose short hood design including the collision posts designs determined using the closed form calculations above must not allow a 65 kip rigid object initially traveling at $30 \mathrm{mph}$ to penetrate into the hood further than 24 inches. Figure 6 depicts the crush sequence of the one-half model. The first state, a, is the undeformed model. The second state, $b$, shows that the hood is the only structural item resisting the penetrating object at 5.25 inches of crush. States $\mathrm{c}$ and $\mathrm{d}$ show the progression of crush from 10.5 inches to 15.25 inches. Between these two states of crush, the interaction effects of the hood and the collision posts become apparent. Finally, states e and f, 20 inches and 24 inches respectively, continue to show how the load is transferred into the system with the collision posts failing at 24 inches of crush and the hood continuing to deform with a decrease in energy absorption.

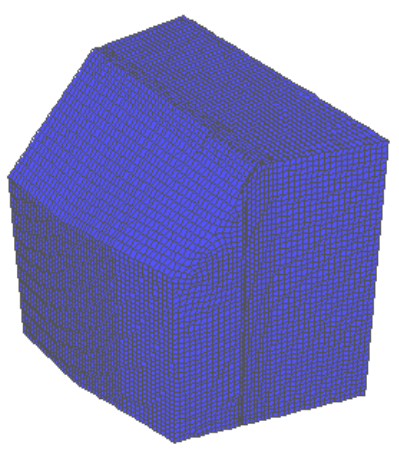

(a)

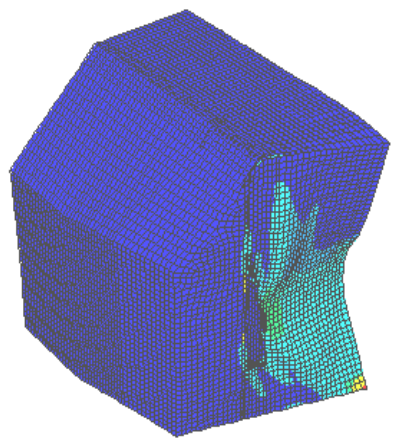

(d)

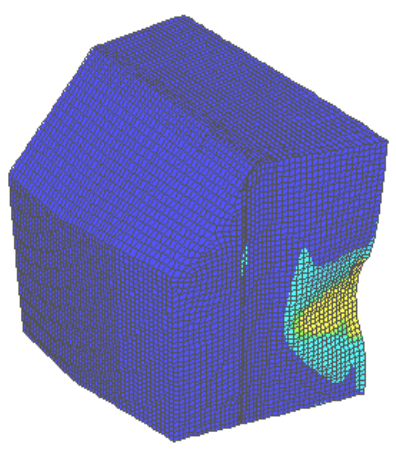

(b)

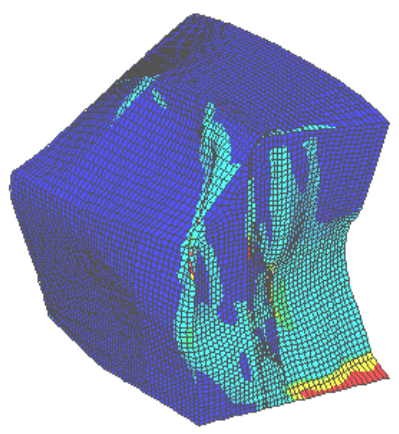

(e)

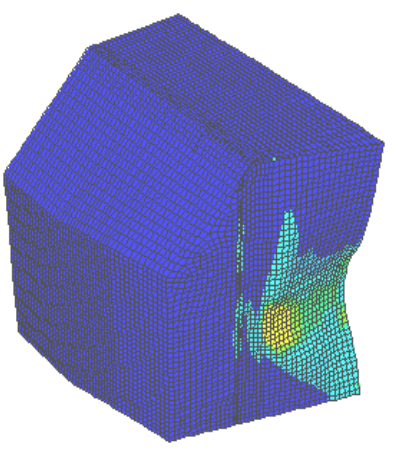

(c)

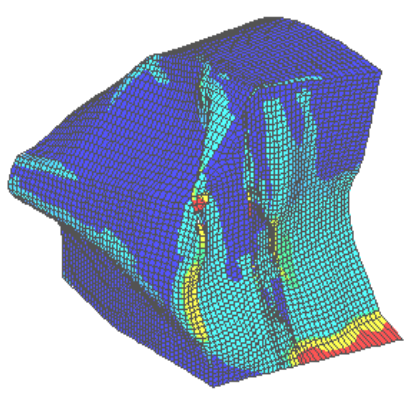

(f)

Figure 6. Crush Sequence of One-Half Model

Figure 7 depicts the force crush response of the hood shown in Figure 6. States $a, b$, and c marked on the graph show the energy absorption due to the hood alone. There is a slight dip in load carrying capacity between states $\mathrm{c}$ and $\mathrm{d}$. The collision posts are struck after 12.5 inches of crush and the load carrying capacity of the complete structural system increases dramatically with a peak load around 2750 kips. This elevated load is maintained over 2.5 inches before it drops to a sustained level of 2250 kips between 16 and 21 inches of crush. Finally the post fails after 21 inches of crush and the load carrying capacity then decreases to the limit of the hood. The two key points determined from the graph are the peak loads, which are enhanced by inertial effects, and the sustainable mean crush load over 24 inches. This value multiplied by the crush distance defined in the description of performance is the total inherent energy absorption capacity of the structural system. To determine if the proposed design meets the desired performance, one compares the original 
collision energy available, 23,450 kip inches, to the capacity of the system $31,200 \mathrm{kip}$ inches. This system is therefore somewhat over-designed with an excess energy absorption capacity of 7,750 kip inches. Provided the failure of the system follows a progressive crush of the hood then post with failure above the sub-base then this design is capable of stopping an object that weighs $65,000 \mathrm{lbs}$ which strikes it with an initial velocity of $34.6 \mathrm{mph}$.

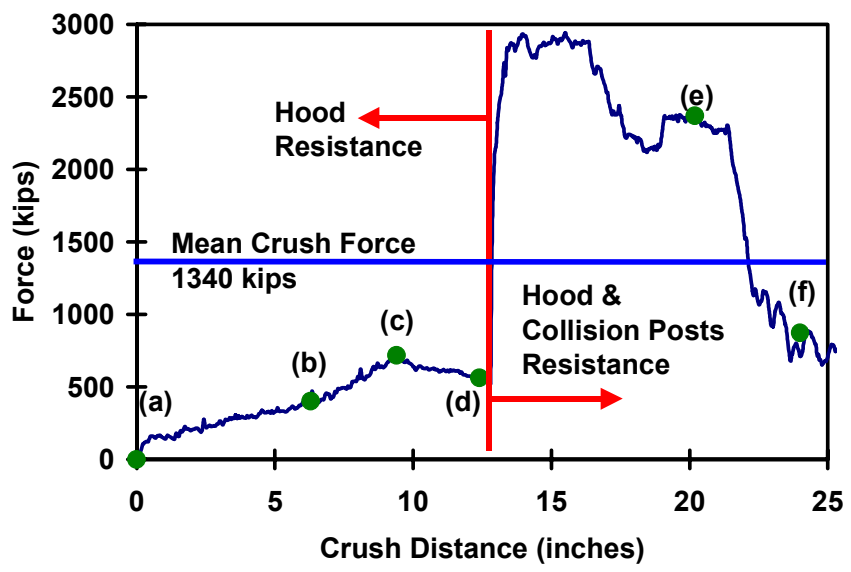

Figure 7. Force Crush Characteristic of Generic Design

\section{SUMMARY AND CONCLUSIONS}

Two methods were used to evaluate the crashworthiness of a locomotive design. The design loads are based upon ultimate or limit load design procedures where the plastic capacity of a section is determined for both torsion and bending. The description of performance establishes a collision scenario where the short hood and collision posts must prevent intrusion of a rigid cylinder into the occupied volume of the cab. The limit load calculations provide information about system response at failure. On a force deflection graph the failure is represented as a single point where the maximum load is obtained. The calculations conducted for the description of performance provide very detailed information about the systems response during the complete impact event including post-buckling response. The force deflection graph is useful to the design engineer because s/he can determine which components of the system provide resistance to the applied load and may help with the refinement of a preliminary design.

One significant difference between the design loads and the description of performance is the direction of load application. The collision post must be able to support the design load applied at $+/-15^{\circ}$ while the description of performance maintains essentially a longitudinal load application. The fully plastic torsional capacity of the section chosen is sufficient to withstand the offset load, but the inclined load appears to be the most critical concern in sizing the section. The concern with the inclined load is the drop in capacity with small changes in load inclination angle. As noted in Figure 3 the drop in load is by a factor close to four. Comparison of the results of the two design approaches show that, for this particular design, the collision posts sized using a limit load approach are somewhat over designed for the description of performance. Since there are inherent differences in the design approach implied by the design loads and the design approach implied by the description of performance, it is to be expected that there will be differences in system response.

\section{ACKNOWLEDGEMENTS}

The authors would like to thank Michael Lorenzen, Manager, Structural Analysis Group, General Motors Electromotive Division (GM/EMD) for discussing crashworthiness analyses approaches. The authors would also like to thank John Punwani, Program Manager, and Claire Orth, Chief Equipment and Operating Practices Division, Office of Research and Development, Federal Railroad Administration for their support.

\section{REFERENCES}

[1] Tyrell, D., Severson, K., Marquis, B., Martinez, E., Mayville, R., Rancatore, R., Stringfellow, R., Hammand, R., Perlman, A. B., "Locomotive Crashworthiness Design Modifications Study," Proceedings of the 1999 IEEE/ASME Joint Railroad Conference, April 13-15, 1999, IEEE Catalog Number 99CH36340, ASME RTD Volume 16, 1999.

[2] Tyrell, D. C., Martinez, E. E., Wierzbicki, T., "Crashworthiness Studies of Locomotive Wide Nose Short Hood Designs," Crashworthiness, Occupant Protection and Biomechanics in Transportation Systems, American Society of Mechanical Engineers, AMD Vol. 237/BED Vol. 45, 1999.

[3] Tyrell, D., Severson, K., Marquis, B., Perlman, A. B., "Simulation of an Oblique Collision of a Locomotive and an Intermodal Container," Crashworthiness, Occupant Protection and Biomechanics in Transportation Systems, American Society of Mechanical Engineers, AMD Vol. 237/BED Vol. 45, 1999.

[4] Pytel, A., Singer, F. A., Strength of Materials, Fourth Edition, Harper \& Row, Publishers, New York, 1987.

[5] Boresi, A. P., Schmidt, R. J., and Sidebottom, O. M., Advanced Mechanics of Materials, Fifth Edition, John Wiley \& Sons, Inc., New York, 1993.

[6] Wierzbicki, T. \& Schneider, F., The Energy Equivalent Flow Stress: Crashworthiness Application, Report No: 15, Impact \& Crashworthiness Laboratory, MIT, 1999.

[7] Hallquist, J. O., LS-DYNA3D User's Manual Version 940, Livermore Software Technology Corporation, California, 1997.

[8] Hibbit, Karlsson and Sorensen, Inc. ABAQUS/Explicit Users Manual, Version 5.8, 1998.

[9] PAMCRASH Manuals, Pam System International, ESI Group Software Product Company, 1996. 